\title{
Tofacitinib
}

\section{JAK-Inhibitor bei rheumatoider Arthritis seit Jahren bewährt}

Der oral einzunehmende Janus-Kinase (JAK)-Hemmer Tofacitinib zeigt bei Patienten mit rheumatoider Arthritis (RA) eine ähnlich gute Wirksamkeit wie Adalimumab. In der EU seit März 2017 zugelassen, liegen aus den USA und anderen Ländern bereits umfangreiche Langzeitdaten vor.

\begin{abstract}
Aus dem Phase III-Studienprogramm ORAL (Oral Rheumatoid Arthritis Phase 3 TriaLs) mit insgesamt fast 4300 Patienten liegen zu Tofacitinib (Xeljanz ${ }^{\circledast}$ ) Daten zur Wirksamkeit auf allen Stufen des Algorithmus der Rheumatherapie [3] vor. In der ORAL Solo-Studie waren bei Patienten mit inadäquatem Ansprechen auf die Vortherapie mit konventionellen krankheitsmodifizierenden antirheumatischen Medikamenten (cDMARDs) im HAQ-DI (Health Assessment Questionnaire - Disability Index) schon nach zweiwöchiger Monotherapie signifikante Verbesserungen im Vergleich zu Placebo erkennbar $(p<0,001)$ [4]. Bis Monat 3 und
\end{abstract}

Deutschland $\mathrm{GmbH}$ darüber hinaus gingen die Einschränkungen noch weiter zurück, berichtete Prof. Dr. med. Torsten Witte, MH Hannover. Tofacitinib ist in Kombination mit Methotrexat (MTX) zugelassen [5]. Es kann aber auch als Monotherapie verordnet werden, wenn MTX ungeeignet ist oder nicht vertragen wird.

\) Die ORAL Solo-Studie zeigte schon nach zweiwöchiger Monotherapie signifikante Verbesserungen

\section{Head-to-Head-Vergleich mit Ada- limumab}

In der Studie ORAL Sync war unter Tofacitinib plus MTX bei Patienten mit unzureichendem Ansprechen auf cDMARDs ebenfalls schon nach zwei Wochen ein Ansprechen erkennbar ( $p \leq 0,001$ vs. Studienbeginn; - Abb. 1) [6]. „Wenn ein Patient anspricht, hält die Wirksamkeit zudem sehr lange an - und hierzu existiert aus Verlängerungsstudien eine wahre Flut von Daten", hob Witte hervor. Bei Respondern belegen sie eine bis zu 90 Monate lang anhaltende Besserung der Symptomatik [7].

》) Tofacitinib ist in Kombination mit Methotrexat zugelassen

In der ORAL Standard-Studie zeigte Tofacitinib eine ähnlich gute Wirksamkeit wie der Tumor-Nekrose-Faktor (TNF)-alpha-Hemmer Adalimumab, beides kombiniert mit MTX [1]. In der aktuell publizierten Phase IIIb/IV-Studie ORAL Strategy mit 1146 Patienten hielt der JAK-Inhibitor erneut dem direkten Vergleich stand [2]. Den primären Endpunkt - eine mindestens $50 \%$ ige Besserung der Symptomatik im American College of Rheumatology (ACR)-Score nach sechs Monaten erreichten $46 \%$ der mit Tofacitinib + MTX und $44 \%$ der mit Adalimumab + MTX behandelten Patienten. Damit konnte die Nicht-Unterlegenheit von Tofacitinib + MTX gegenüber Adalimumab + MTX mit statistischer Signifikanz nachgewiesen werden $(p<0,0001)$.

\section{Dr. Matthias Herrmann}

\section{Literatur}

1. van Vollenhoven R et al (2012) N Engl J Med 367:508-519

2. Fleischmann R et al (2017) Lancet 2017:

3. Krüger K et al (2012) Z Rheumatol 71:592-603

4. Fleischmann R et al (2012) N Engl J Med 367:495-507

5. Fachinformation Xeljanz ' Stand Mai 2017

6. Kremer J et al (2013) Ann Intern Med 159:253-261

7. Wollenhaupt $J$ et al. Jahrestagung des American College of Rheumatology (ACR) und der Association for Rheumatology Health Professionals (ARHP), Washington, DC, 11.-16. November 2016, Poster 1647

rheuma plus $2017 \cdot 16: 120$ https://doi.org/10.1007/s12688017-0138-y (c) Springer-Verlag GmbH Austria 2017 